\title{
Double-autoionization decay of resonantly excited single-electron states
}

\author{
M Ya Amusia †े|, V A Kilin $¥$, A Ehresmann $\neq$, H Schmoranzerł \\ and K-H Schartner§ \\ t Institut für Theoretische Physik, Johann-Wolfgang-Goethe-Universität, D.6000 Frank- \\ furt/Main 11, Federal Republic of Germany \\ \$ Fachbereich Physik, Universität Kaiserslautern, D-6750 Kaiserslautern, Federal Republic \\ of Germany \\ 81. Physikalisches Institut, Justus-Liebig-Universitāt, D-6300 Giessen, Federal Republic \\ of Germany
}

Received 24 August 1992, in final form 30 December 1992

\begin{abstract}
Perturbation theory in the lowest non-vanishing order in interelectron interaction has been applied to the theoretical investigation of double-ionization decays of resonantly excited single-electron states. The formulae for the transition probabilities were derived in the LS coupling scheme, and the orbital angular momentum and spin selection rules were obtained. In addition to the formulae, which are exact in this order, three approximate expressions, which correspond to illustrative model mechanisms of the transition, were derived as limiting cases of the exact ones. Numerical results were obtained for the decay of the resonantly excited $\mathrm{Kr}, 3 \mathrm{~d}^{-1} \mathrm{Sp}\left[{ }^{2} \mathrm{P}\right]$ state which demonstrated quite clearly the important role of the interelectron interaction in double-ionization processes. On the other hand, the results obtained show that low-energy electrons can appear in the photoelectron spectrum below the ionization threshold of the 3d shell. As a function of the photon frequency, the yield of these low-energy electrons is strongly amplified by the resonant transition of the $3 d$ electron to $5 p$ (or to other discrete levels), acting as an intermediate state, when the photon frequency approaches that of the transition.
\end{abstract}

\section{Introduction}

In recent years, a number of new experimental data have shown the manifestation of the correlative effects in photoelectron and fluorescence spectra. For example, in photoionization cross sections of subvalence shells of rare gases as well as in excitation cross sections of the corresponding satellite states, strong structures were observed in an energy interval of a few eV close to their respective thresholds (Becker et al 1986a, b, 1988, Hall et al 1989, 1990, Wills et al 1990a, b, Schartner et al 1988, 1989, 1990, Schmoranzer et al 1990, Ehresmann et al 1992). These structures are mostly due to the decay of resonantly doubly-excited atomic states.

Similarly, the decay of the $\operatorname{ArI} 3 p^{-1} n s, n d$, the $K_{r}{ }^{3} d^{-1} n p$ and the $X e 14 d^{-1} n p$ resonances was found to yield new features in photoelectron, Auger and fluorescence spectra (Eberhardt et al 1978, Aksela et al 1984, 1986a, b, 1989, Becker et al 1986a, b, Lindle et al 1987, Svensson et al 1988, von Raven et al 1990, Lablanquie and Morin

|| Permanent address: A F Ioffe Physical Technical Institute of the Academy of Science, St Petersburg, Russia. I Permanent address: Department of Mathematics-1, Tomsk Polytechnic Institute, 634004 Tomsk, Russia. 
1991, Hall et al 1992, Schmoranzer et al 1991). Transitions from these resonantly excited atomic states to lower lying ionic states occur in one, two or more consecutive steps. Some of these transitions may be described in the frame of single-particle approximations, for example by using the 'spectator' model. The others are purely correlative transitions and essentially of many-electron nature. One example of such a transition is the decay of the resonantly excited $\mathrm{Kr} 13 \mathrm{~d}^{-1} 5 \mathrm{p}$ state ending in the simultaneous ejection of two electrons. This decay may be considered as the second step of a two-step single-photon double-ionization process.

Theoretical investigations of this specific process were so far limited mainly by the 'shake-off' approximation (Aksela et al 1989) or by the two-step cascade model (von Raven et al 1990). However, a more general approach exists, i.e. the perturbation theory in interelectron interaction, which has been applied, e.g., to the description of the double-Auger decay of the 1 s vacancy in Ne (Amusia et al 1985). The same approach is used here to describe theoretically a double-Auger-like autoionization decay of the resonantly excited state, which we call the double-electron autoionization process.

The formulae derived are obtained in the lowest non-vanishing order of perturbation theory by using the $L S$ coupling scheme. In addition to the formulae which are correct in this order, three approximate expressions are presented, which follow from the correct ones as limiting cases. They are useful for simple estimations of the transition probabilities and for establishing the real physical mechanisms of the above process.

Numerical results will be obtained for the transitions $3 d^{-1} 5 p\left[{ }^{1} P\right] \rightarrow 4 s^{-2}\left[{ }^{1} S\right]$, $4 s^{-1} 4 \mathrm{p}^{-1}\left[{ }^{1,3} \mathrm{P}\right]$ in $\mathrm{Kr}$, which are of particular interest at present for the explanation of recent experimental data obtained by photon-induced fluorescence spectroscopy (PIF) (Schmoranzer et al 1991).

\section{Theoretical base}

Let us consider the transition from the resonantly excited single-electron atomic state to the doubly ionized final state (see, e.g., figure 1). The energy $\Delta E$ of the transition is equal to the difference between the energy of the double-vacancy ionic state $E_{f}^{\text {ion }}$ and the atomic excited state $E_{i}$. This excess energy $\Delta E$ may be distributed continuously between two outgoing electrons. In this sense the double-electron autoionization process is similar to the double-Auger decay of a single atomic vacancy.

The total transition probability (or total width) for the decay of the resonance is equal to the integral

$$
\Gamma=\int_{0}^{\Delta E} \gamma(\varepsilon) d \varepsilon
$$

where $\gamma(\varepsilon)$ is the density of probability to detect one ejected electron with the energy $\varepsilon$, while the energy of the second electron is equal to $(\Delta E-\varepsilon)$. The distribution function in equation (1) is defined by equation (2) $\uparrow$ :

$$
\gamma(\varepsilon)=2 \pi|M(\varepsilon)|^{2} \delta\left(E_{1}-E_{f}\right) \text {. }
$$

The amplitude $M(\varepsilon)$ of the radiationless transition between the initial and final states described by their exact multielectron wavefunctions $\psi_{s}$ and $\psi_{f}$, respectively, is given

† Atomic units are used throughout this paper. 


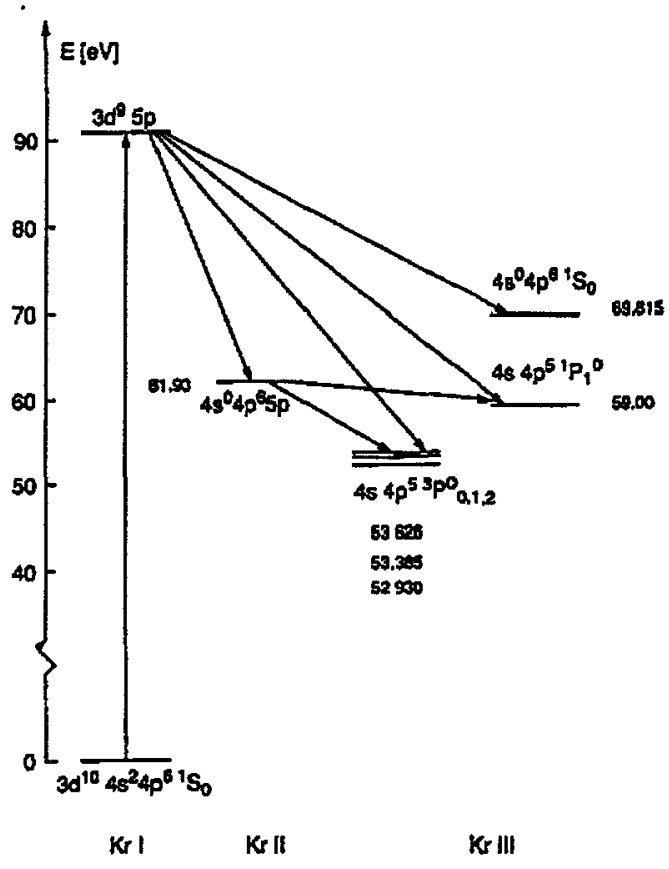

Figure 1. The energy levels (Moore 1971) and possible pathways under consideration of the double-ionization decay of the resonantly excited $\mathrm{Kr}$ I $3 d^{-1} 5 p$ state.

by their overlap integral:

$$
M=\left\langle\psi_{l} \mid \psi_{f}\right\rangle
$$

Usually, in simple Auger decays, the first-order perturbative approach is sufficient and the transition from the initial instantly created vacancy to the final state with two vacancies and an Auger electron proceeds due to the interelectron interaction $V$,

$$
V=\frac{1}{2} \sum_{p, q=1}^{N} \frac{1}{\left|r_{p}-r_{q}\right|}
$$

where $N$ is the number of atomic electrons.

In the specific case of interest here, the initial state has a vacancy in the $i$ shell and an excited electron on the discrete level $n$. The final state has two vacancies (holes) $f_{1}$ and $f_{2}$, while two electrons $e_{1}$ and $e_{2}$ are in the continuum. As $V$ (equation (4)) is a two-body operator, it can only describe, in the first-order perturbation theory, an Auger-like decay of the vacancy $i$ with the electron $n$ being a spectator of the autoionization decay of the initial state with emission of a single electron. Thus in order to reach the considered final state, the interelectron interaction must be included at least in the second order. This means that the transition under consideration is a real correlative process, in which no less than three electrons participate actively. The best one-electron states are determined in Hartree-Fock approximation by the Hamiltonian $\hat{H}^{\mathrm{HF}}$. Thus the correlative interaction is determined by the difference

$$
\hat{V}_{\text {corr }}=\hat{H}-\hat{H}^{H F}
$$

$\hat{H}$ being the total atomic Hamiltonian. 
The perturbation theory approach is used in this paper. The contribution of the lowest non-vanishing order to the amplitude $M$ of the considered process is given by the sum of many-body diagrams depicted in figure 2. The usual notation of the many-body theory is used. Namely, the line with an arrow to the left (right) represents a vacancy (continuum electron), a line with a double arrow stands for an electron on a discrete excited level, while the wavy line denotes the Coulomb interelectron interaction.

The corresponding analytical expressions for these partial amplitudes have the following form

$$
\begin{aligned}
& M_{1}=\sum_{k}\left\langle k e_{1}|u| f_{1} f_{2}\right\rangle\left\langle e_{2} i|u| n k\right\rangle E_{k e_{1} s_{1} s_{2}}^{-1} \\
& M_{2}=\sum_{k}\left\langle k e_{1}|u| f_{2} n\right\rangle\left\langle e_{2} i|u| f_{1} k\right\rangle E_{k e_{2} f_{2} n}^{-1} \\
& M_{3}=\sum_{k}\left\langle k e_{1}|u| f_{1} n\right\rangle\left\langle i e_{2}|u| f_{2} k\right\rangle E_{k e_{1} n f_{1}}^{-1} \\
& M_{4}=\sum_{k}\left\langle k e_{2}|u| f_{2} f_{1}\right\rangle\left\langle e_{1} i|u| n k\right\rangle E_{k e_{2} f_{1} f_{2}}^{-1} \\
& M_{5}=\sum_{k}\left\langle k e_{2}|u| f_{2} n\right\rangle\left\langle e_{1}|u| f_{1} k\right\rangle E_{k e_{2} s_{2} n}^{-1} \\
& M_{6}=\sum_{k}\left\langle k e_{2}|u| n f_{1}\right\rangle\left\langle i e_{1}|u| f_{2} k\right\rangle E_{k e_{2} n f_{3}}^{-1} \\
& M_{7}=\sum_{k}\left\langle k i|u| f_{1} f_{2}\right\rangle\left\langle e_{1} e_{2}|u| n k\right\rangle E_{k i i_{1} s_{2}}^{-1} \\
& M_{8}=\sum_{k}\left\langle k i|u| n f_{2}\right\rangle\left\langle e_{2} e_{1}|u| f_{1} k\right\rangle E_{k i j_{2} n}^{-1} \\
& M_{9}=\sum_{k}\left\langle k i|u| n f_{1}\right\rangle\left\langle e_{1} e_{2}|u| f_{2} k\right\rangle E_{k i v_{1}}^{-1} .
\end{aligned}
$$
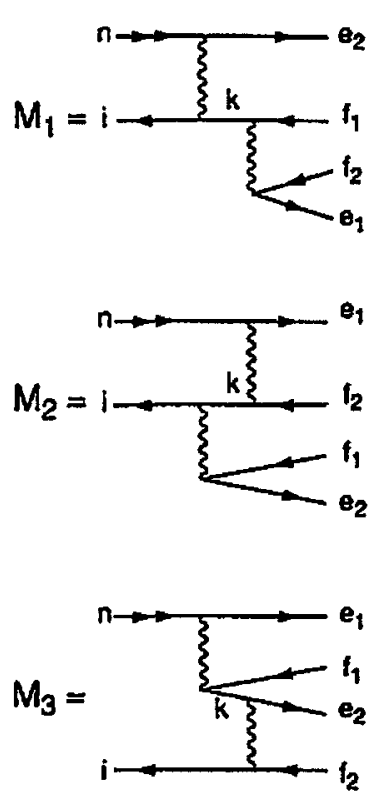
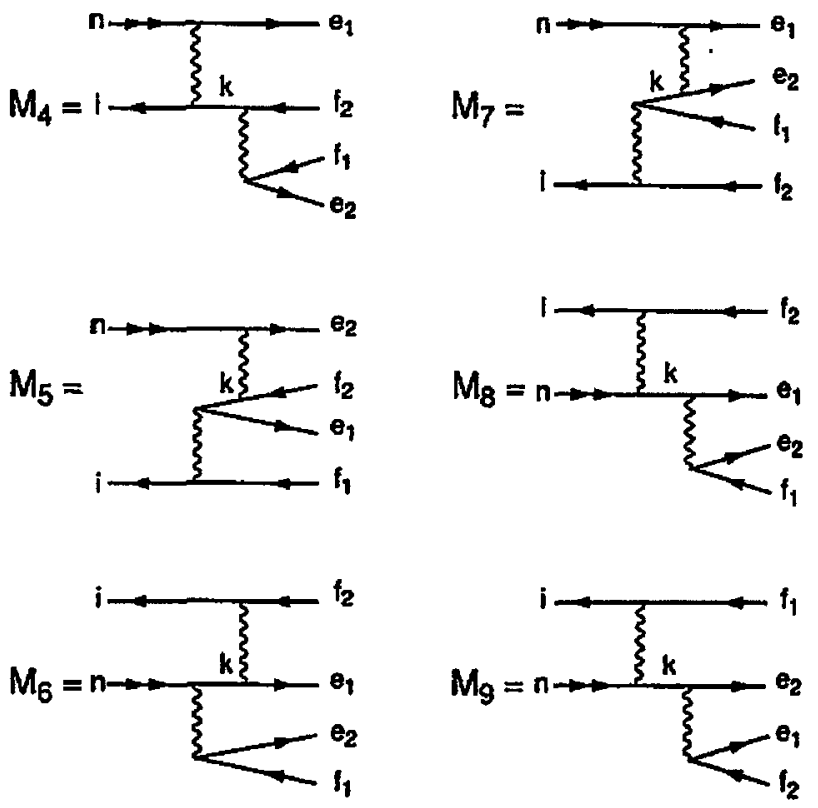

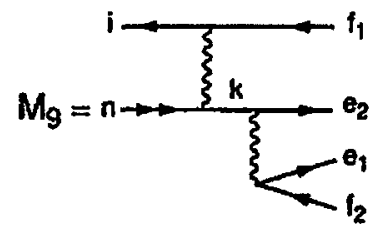

Figure 2. The lowest non-vanishing-order perturbation theory diagrams of the transition $\left(\right.$ in $\left.\rightarrow f_{1} f_{2}+e_{1}+e_{2}\right)$. A line with an arrow (two arrows) to the right describes an electron continuum (discrete excited) state, while a line with an arrow to the left corresponds to a hole state; a wavy line denotes interelectron Coulomb interaction. 
Here $E_{k l m n}=\varepsilon_{k}+\varepsilon_{1}-\varepsilon_{m}-\varepsilon_{n}$ is the intermediate state energy, $\varepsilon_{j}$ denotes the energies of the single-electron $\mathrm{HF}$ states, and $\langle k l|u| m n\rangle$ stands for a combination of the direct and exchange Coulomb matrix elements:

$$
\langle k|| u \mid m n\rangle=\left\langle k l\left|r_{12}^{-1}\right| m n\right\rangle-\left\langle k l\left|r_{12}^{-1}\right| m n\right\rangle .
$$

The sum over $k$ in equation (6) includes both the hole and discrete excited levels and the integration over the continuum states. If $E_{k j m n}$ can vanish while $k$ belongs to the continuum, the following integration formula is used:

$$
\int \frac{f(x)}{x-x_{0}+\mathrm{i} \delta} \mathrm{d} x=P \int \frac{f(x)}{x-x_{0}} \mathrm{~d} x \mp \mathrm{i} \pi f\left(x_{0}\right)
$$

where $P$ denotes the principal value of the integral.

The final expressions for partial amplitudes $M_{\alpha}, \alpha=1, \ldots, 9$, suitable for real calculations, are presented in the appendix. They are obtained under the assumption that the $L S$ coupling scheme for both the initial and final states is valid, so that their orbital angular momenta and spins are defined:

$$
\begin{aligned}
& \psi_{1}=|\operatorname{in}(L S)\rangle \\
& \psi_{f}=\left|f_{1} f_{2}\left(L_{1} S_{1}\right) e_{1} e_{2}\left(L_{2} S_{2}\right) L^{\prime} S^{\prime}\right\rangle
\end{aligned}
$$

In evaluating equation (6), at first the integration over angular variables was performed and then the summing over spin variables. Finally it was summed over all possible projections of orbital and spin momenta.

Below we shall refer to equation (6) and (A.1-9) as the exact formulae, of course having in mind their perturbative nature. These exact formulae (A.1-9) automatically lead to the orbital angular and spin momenta selection rules for the transition under consideration. The selection rules contain the information about both the angular momenta of electrons (vacancies) involved in the transition and the intermediatecoupling momenta $L_{1} S_{1}, L_{2} S_{2}$ in equation (9).

\section{Approximations}

Even at a first glance, the exact formulae (equation (6)) and (A.1-9) look rather complex. Therefore it is difficult to use them for estimations of the transition probabilities. Furthermore their structure masks to some extent the most important real physical mechanism (if such a dominant mechanism exists) responsible for a given transition.

At least three special cases may be distinguished, in which the estimation of probabilities is considerably simplified.

\subsection{Shake off model}

Suppose that the main contribution to the total amplitude $M$ is due to some large Coulomb matrix elements in equation (6). Normally the largest ones are the matrix elements, which include two or more identical single-electron wavefunctions. Let us 
sum, following Amusia and Cherepkov (1975), the partial amplitudes $M_{1}(k=i), M_{5}$ ( $\left.k=f_{2}\right)$ and $M_{6}\left(k=f_{1}\right)$. Then we have, after factoring out,

$$
\begin{gathered}
\left\langle i e_{1}|u| f_{1} f_{2}\right\rangle\left[\left\langlee_{2} i\left[u|n i\rangle-\left\langle e_{2} f_{1}|u| n f_{1}\right\rangle-\left\langle e_{2} f_{2}|u| n f_{2}\right\rangle\right] /\left(\varepsilon_{2}-\varepsilon_{n}\right)\right.\right. \\
=\left\langle i e_{1}|u| f_{1} f_{2}\right\rangle\left\langle e_{2}\left|F^{(i)}-F^{\left.U_{1} f_{2}\right)}\right| n\right\rangle\left(\varepsilon_{2}-\varepsilon_{n}\right) .
\end{gathered}
$$

Here $F^{(l)}$ is by definition the lowest-order correction to the atomic self-consistent field due to the absence of an electron on the level $i$ in the initial state; $F^{\left(\delta_{1} f_{2}\right)}$ is a similar correction due to the presence of the holes $f_{1}$ and $f_{2}$ in the final state; $\varepsilon_{2}$ and $\varepsilon_{n}$ are the single-particle energies for $e_{2}$ and $n_{3}$ respectively. Now let $|\tilde{n}\rangle$ and $\left|\tilde{e}_{2}\right\rangle$ denote the solutions of the single-electron Hartree-Fock equations

$$
\begin{aligned}
& \hat{H}_{i}^{(\mathrm{HF})}|\tilde{n}\rangle=\tilde{\varepsilon}_{n}|\tilde{n}\rangle \\
& \hat{H}_{\delta_{1} \sqrt{2}_{2}}^{(\mathrm{HF})}\left|\tilde{e}_{2}\right\rangle=\tilde{\varepsilon}_{2}\left|\tilde{e}_{2}\right\rangle
\end{aligned}
$$

where $\hat{H}_{i}^{(\mathrm{HF})}$ and $\hat{H}_{f_{3}}^{(\mathrm{HF})}$ are the Hartree-Fock Hamiltonians of ions, which may be obtained from that for an atom $H^{(\mathrm{HF})}$ by excluding the electrons in the states $i$ and $f_{1}, f_{2}$, respectively. Then the second factor in equation (10) may be transformed into the following form:

$$
\left\langle e_{2}\left|F^{(i)}-F^{\left(\varepsilon_{1} f_{2}\right)}\right| n\right\rangle /\left(\varepsilon_{2}-\varepsilon_{n}\right) \approx\left\langle e_{2}\left|\hat{H}_{S_{2} \sqrt{2}_{2}}^{(\mathrm{HF})}-\hat{H}_{1}^{(\mathrm{HF})}\right| n\right\rangle /\left(\varepsilon_{2}-\varepsilon_{n}\right) \approx\left\langle\tilde{e}_{2} \mid \tilde{n}\right\rangle .
$$

Here $\left\langle\tilde{e}_{2} \mid \tilde{n}\right\rangle$ is the overlap integral of the excited-electron wavefunction $|\tilde{n}\rangle$, obtained in the field of the initial vacancy $i$ and the continuum-electron wavefunction $\left|\tilde{e}_{2}\right\rangle$, defined in the field of two vacancies $f_{1}$ and $f_{2}$, i.e. in the field of the residual ion.

Finally, we obtain a rather simple formula for the amplitude, which corresponds to the so-called 'shake off' approximation:

$$
M_{\text {sh }}=\left\langle i e_{1}|u| f_{1} f_{2}\right\rangle\left\langle\tilde{e}_{2} \mid \tilde{n}\right\rangle .
$$

A similar formula may be easily obtained by combining contributions of other partial amplitudes $M_{4}(k=i), M_{2}\left(k=f_{1}\right)$ and $M_{3}\left(k=f_{2}\right)$ :

$$
M_{\mathrm{sb}}=\left\langle i e_{2}|u| f_{1} f_{2}\right\rangle\left\langle\tilde{e}_{1} \mid \tilde{n}\right\rangle \text {. }
$$

Since the overlap integral $\left\langle\tilde{e}_{2} \mid \tilde{n}\right\rangle$ is not only proportional to the Coulomb matrix element but also inversely proportional to $\left(\varepsilon_{2}-\varepsilon_{n}\right)$, one may expect a large contribution of the 'shake off mechanism in cases when one of the outgoing electrons has the lowest possible kinetic energy, while the Coulomb transition matrix element $\left\langle i e_{1}|u| f_{1} f_{2}\right\rangle$ is large.

\subsection{Two-step or cascade process}

The cascade mechanism of the transition in $\rightarrow f_{1} f_{2}+e_{1}+e_{2}$ is possible, if on the way from the initial atomic state with the energy $E_{i}$ to the final ionic state of energy $E_{f}^{\text {ion }}$ there is at least one real ionic state through which the decay may proceed if allowed by the spin and orbital angular momentum conservation laws. In this case, the energy denominator in one of the partial amplitudes (equation (6), figure 1) becomes small or even is equal to zero. Thus the distribution of energy between the two outgoing electrons is close to that of a real multi-step process. In order to treat the singularity arising from the vanishing denominator correctly, one has to take into account the total width $\Gamma_{k}$ of this intermediate ionic state.

Assume that such a situation occurs in reality, e.g. in the partial amplitude $M_{5}$, when the intermediate single-electron state $k=k_{0}$, the hole state $f_{1}$ and the excitedelectron state $n$ form jointly the real intermediate electron configuration $\left(k_{0} f_{1} n\right)$ of 
energy $E_{k_{0} f_{1} n}$, where $E_{f}^{\text {lon }}<E_{k_{0} f_{1} n}<E_{i}$. Then points of singularity in the $\left(e_{1}, e_{2}\right)$ energy distribution will be found, where the energy denominator is small or even tends to zero. In this case, the contribution to the amplitude $M_{5}\left(k=k_{0}\right)$ must be modified by introducing the width $\Gamma_{k}$ of the state $k$ in the denominator:

$$
M_{5}\left(k=k_{0}\right)=\frac{\left\langle k_{0} e_{2}|u| f_{2} n\right\rangle\left(i e_{1}|u| f_{1} k_{0}\right\rangle}{\Delta \varepsilon+i\left(\Gamma_{k} / 2\right)}=M_{\mathrm{ts}}
$$

This expression corresponds to an infinite sum of diagrams which accounts for the interaction between the outgoing electron $e_{2}$ and the vacancy $f_{2}$, including the possibility for them to go back to the states $n$ and $k$, respectively. Equation (15) describes the consecutive two-step transition. The first step is the Auger-like transition (in $\rightarrow f_{1} k_{0} n+$ $e_{1}$ ) with an excited electron $n$ as a 'spectator' and resulting in ejection of the first-step Auger electron $e_{1}$ with the energy $\varepsilon_{1}=E_{1}-E_{k_{0} f_{1} n}$. The second step is the decay of an intermediate autoionization state $\left(k_{0} f_{1} n \rightarrow f_{1} f_{2}+e_{2}\right)$. Now the vacancy $f_{1}$ plays the role of a 'spectator' and the second-step electron $e_{2}$ with the energy $\varepsilon_{2}=E_{k_{0} f_{n} n}-E_{f}^{\text {ion }}$ is ejected. As a result, the normally smooth curve $\gamma(\varepsilon)$ acquires prominent peaks at the energy positions $\varepsilon=\varepsilon_{1}$ and $\varepsilon=\varepsilon_{2}$. Similarly, not only two, but many peaks in the electron spectra can be present.

\subsection{Resonance in continuzum}

The energy denominator in a partial amplitude may be equal to zero also when the intermediate state $k=e_{k}$ belongs to the continuum. Such a situation is possible, e.g., in the partial amplitude $M_{7}$. According to equation (8), the contribution just of this continuum state $k=e_{k}$ to the total amplitude $M$ is given by the expression

$$
M_{7}\left(k=e_{k}\right)=\mp i \pi\left\langle e_{k} i|u| f_{1} f_{2}\right\rangle\left\langle e_{1} e_{i}|u| n e_{k}\right\rangle \equiv M_{\mathrm{rc}} \text {. }
$$

This may also be the dominant contribution, if the Coulomb matrix elements in equation (16) are large. In this case, one may consider the transition in $\rightarrow f_{1} f_{2}+e_{1}+e_{2}$ as proceeding by two steps. The first one is an Auger-like decay in $\rightarrow f_{1} f_{2} n \rightarrow e_{k}$ with an excited electron $n$ as a 'spectator', which results in ejection of an intermediate electron $e_{k}$ with energy $\varepsilon_{k}$. The second step is the inelastic scattering of this electron $e_{k}$ by the electron $n$ at the discrete excited level. As a result the latter acquires the additional energy and leaves the atom. The scattered electrons may change their orbital momenta and spin in the course of the interaction.

In the real transitions, as will be demonstrated by the results of numerical calculations, all the simplifying mechanisms presented above contribute to the total amplitude. However, the contributions of the remaining intermediate states $k$, both discrete and continuous ones, which are not taken into account by the simplified approaches described above, may be (and quite frequently are) significant. The interference of the various amplitudes may result in both increase and decrease as compared to the results of the simplified calculations. In some cases, the model estimations of the probabilities may even be misleading.

\section{Results and discussion}

Here we present the main results of numerical calculations for the transitions from the resonantly excited $\mathrm{KrI} 3 \mathrm{~d}^{-1} 5 \mathrm{p}\left[{ }^{1} \mathrm{P}\right]$ state to the (i) $\mathrm{Kr}$ III $4 \mathrm{~s}^{-2}\left[{ }^{-1} \mathrm{~S}\right]$, (ii) 
$\mathrm{Kr}$ III $4 s^{-1} 4 \mathrm{p}^{-1}\left[{ }^{1} \mathrm{PI}\right.$ and (iii) $\mathrm{Kr} \mathrm{IIr} 4 \mathrm{~s}^{-3} 4 \mathrm{p}^{-1}\left[{ }^{3} \mathrm{P}\right]$ states of the doubly charged ion (figure 1). These transitions are the most intense ones which lead to $\mathrm{Kr}$ III states observable by pIFs. In all three cases, two electrons are ejected at the end taking away the energy of the transition. There is one exclusively correlative channel, i.e. the direct transition $3 \mathrm{~d}^{-1} 5 \mathrm{p}\left[{ }^{1} \mathrm{P}\right] \rightarrow 4 \mathrm{~s}^{-2}+e_{1}+e_{2}$, while the transitions $3 \mathrm{~d}^{-1} 5 \mathrm{p}\left[{ }^{1} \mathrm{P}\right] \rightarrow$ $4 s^{-1} 4 \mathrm{p}^{-1}\left[{ }^{1,3} \mathrm{P}\right]+e_{1}+e_{2}$ may proceed, in addition to the direct path, by two-step processes

$$
\begin{aligned}
3 \mathrm{~d}^{-1} 5 \mathrm{p}\left[{ }^{-1} \mathrm{P}\right] & \rightarrow 4 \mathrm{~s}^{-2} 5 \mathrm{p}\left[{ }^{2} \mathrm{P}\right]+e_{1} \\
& \stackrel{L}{\longrightarrow} 4 \mathrm{~s}^{-1} 4 \mathrm{p}^{-1}\left[{ }^{1,3} \mathrm{P}\right]+e_{2} .
\end{aligned}
$$

The aim of our calculations is to obtain the probabilities of the various channels and to find, if they exist at all, the dominant mechanisms of these transitions. The computer codes 'ATOM' (Chernysheva and Amusia 1983) are used for numerical calculations of the energy levels, wavefunctions, amplitudes and distribution functions.

The energies of the initial $3 d^{-1} 5 p\left[{ }^{1} P\right]$, intermediate $4 s^{-2} 5 p\left[{ }^{2} P\right]$ and final ionic $4 \mathrm{~s}^{-2}\left[{ }^{-1} \mathrm{~S}\right], 4 \mathrm{~s}^{-1} 4 \mathrm{p}^{-1}\left[{ }^{1},{ }^{3} \mathrm{P}\right]$ states, obtained within the single-configuration non-relativistic HF approximation, have correct relative positions, but the absolute values are not in good agreement with the experimental data. Therefore we used experimental energy values (Moore 1971) in our calculations of the decay probabilities.

The wavefunctions of the ejected electrons are obtained separately by calculating them in the self-consistent field of the corresponding doubly charged ion state. So we neglect here the interaction between the outgoing electrons. This approximation seems to be reasonable because both the experimental data and theoretical estimations show the highest probability for the situation, when one of the outgoing electrons is fast while the other is slow. Note that the above wavefunctions of ejected electrons were calculated by taking into account that the field depends on the term of the double-hole state, e.g. by considering the ${ }^{1} \mathrm{P}$ and ${ }^{3} \mathrm{P}$ terms of the $4 \mathrm{~s}^{-1} 4 \mathrm{p}^{-1}$ state. As was shown earlier (Amusia and Cherepkov 1975), the use of such electron wavefunctions is equivalent to the inclusion of an infinite sequence of the diagrams of the so-called random phase approximation with exchange (RPAE). For the summation over intermediate single-particle states $k$ in equation (6), we use the hole wavefunctions of the $\mathrm{Kr}$ ground state and the wavefunctions of the electron, excited from the specific atomic shell just as it is represented in the diagram under consideration, i.e. in the 'frozen' field of $(N-1)$ remaining atomic electrons. The continuous wavefunctions of the intermediate states $k$ in equation (6) are single-particle wavefunctions calculated in the term-dependent $\mathrm{HF}$ approximation with the term 'P. In this case, a definite part of the RPAE diagram contributions is taken into account also (Amusia and Cherepkov 1975).

The number of intermediate discrete and continuum states involved in the summation (integration) over $k$ in equation (6) was established by preparatory calculations: 4 discrete and 39 continuum states were used for each value of angular momentum $\boldsymbol{l}_{\boldsymbol{k}}$. Distribution functions $\gamma(\varepsilon)$ were calculated at 9 to 25 points of energy $\varepsilon$, depending on whether the curve is smooth or has a singular structure. The probabilities were obtained by numerical integration of $\gamma(\varepsilon)$ according to equation (1).

\subsection{Transition $3 d^{-3} 5 p\left[{ }^{1} P\right] \rightarrow 4 s^{-2}[3 S]+e_{1}+e_{2}$}

The energy of this transition is equal to $91.20-69.82=21.38 \mathrm{eV}$. According to the selection rules, the transition may result in ejection of different electron pairs $\left(e_{1}, e_{2}\right)$ 
having non-limited orbital angular momenta $(1,1+1), 1=0,1,2 \ldots$ However, the total orbital angular momentum and spin of the electron pair is restricted to $L_{2}=1, S_{2}=0$. We shall see below that the probability to emit electrons having large angular momenta is decreasing significantly with increasing 1 , so that it is sufficient to take into account the first few values of 1 , up to $1=6$, only.

As was mentioned above, the intermediate states between $3 d^{-1} 5 \mathrm{p}\left[{ }^{1} \mathrm{P}\right]$ and $4 \mathrm{~s}^{-2}\left[{ }^{1} \mathrm{~S}\right]$ may be only virtual in our approximation. Figure 3 shows the distribution functions $\gamma_{1,1+1}(\varepsilon)$ of the energy of an outgoing electron (the left symmetrical half only). The upper curve is the sum of $\gamma_{1,1+1}(\varepsilon), 1=1, \ldots, 6$, and corresponds to the total distribution function $\gamma(\varepsilon)$. These curves have a characteristic U-like shape. This means that if there is enough energy for emitting two electrons, then one is predominantly slow and the other is fast. The integral contributions of different electron pairs to the total probability of the transition are presented in table 1 . The main contribution comes from the ejection of a $(p, d)$ electron pair. To illustrate the contributions of the 'model' mechanisms, we present in table 2 the intermediate numerical results for partial amplitudes $M_{\alpha}$, $\alpha=1,2, \ldots, 9$, for the transition $3 \mathrm{~d}^{-1} 5 \mathrm{p}\left[{ }^{1} \mathrm{P}\right] \rightarrow 4 \mathrm{~s}^{-2}\left[{ }^{1} \mathrm{~S}\right]+e_{p}+e_{d}$ at three essentially

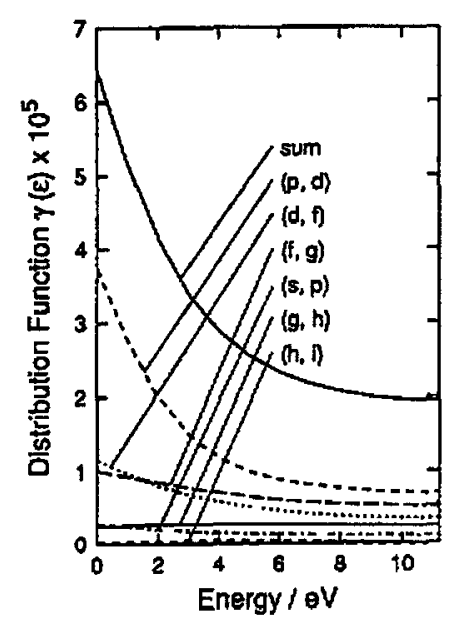

Fgare 3. The distribution functions $\gamma_{b+1}(\varepsilon)$ (left symmetrical half) of the energy $\varepsilon$ of an outgoing electron in the transition $3 d^{-1} 5 p\left[{ }^{1} \mathrm{P}\right] \rightarrow 4 s^{-2}[1 S]+e_{1}+e_{2}$. The upper solid line corresponds to the total distribution function $\gamma(\varepsilon)$ of the transition.

Table 1. Integral contributions $\Gamma_{\text {reser }}$ of different pairs $(h, l+1)$ to the total transition probability of $3 d^{-1} 5 p\left[{ }^{1} \mathrm{P}\right] \rightarrow 4 \mathrm{~s}^{-2}\left[{ }^{1} \mathrm{~S}\right]$, in $10^{-4} \mathrm{eV}$.

\begin{tabular}{llc}
\hline $\begin{array}{l}\text { Electron pair } \\
\left(e_{1}, e_{2}\right)\end{array}$ & $\Gamma_{{\text {1 } \varepsilon_{2}}}\left(10^{-4} \mathrm{eV}\right)$ & Fraction $(\%)$ \\
\hline$(\mathrm{s}, \mathrm{p})$ & 0.29 & 5 \\
$(\mathrm{p}, \mathrm{d})$ & 2.43 & 43 \\
$(\mathrm{~d}, \mathrm{f})$ & 1.06 & 19 \\
$(\mathrm{f}, \mathrm{g})$ & 1.27 & 23 \\
$(\mathrm{~g}, \mathrm{~h})$ & 0.50 & 9 \\
$(\mathrm{~h}, \mathrm{i})$ & 0.05 & $<1$ \\
$\Sigma \Gamma_{c_{1} c_{2}}$ & 5.60 & 100 \\
\hline
\end{tabular}


Table 2. Partial amplitudes $M_{a}$, total amplitude $M$ and model amplitudes $M_{t h}, M_{t e,} M_{x e}$ of the transition $3 \mathrm{~d}^{-1} 5 \mathrm{p}\left[{ }^{-} \mathrm{P}\right] \rightarrow 4 \mathrm{~s}^{-2}\left[{ }^{1} \mathrm{~S}\right]+e_{\mathrm{p}}+e_{\mathrm{a}}$ at different electron energies $\varepsilon_{p}$.

\begin{tabular}{|c|c|c|c|c|c|c|}
\hline \multirow[b]{2}{*}{$\alpha$} & \multicolumn{2}{|c|}{$\varepsilon_{\mathrm{s}}=0.0136 \mathrm{eV}$} & \multicolumn{2}{|c|}{$\varepsilon_{\mathrm{p}}=10.69 \mathrm{eV}$} & \multicolumn{2}{|c|}{$e_{p}=21.3663 \mathrm{eV}$} \\
\hline & $M_{\alpha}$ & Main contribution $(k)$ & $M_{\alpha}$ & Main contribution $(k)$ & $M_{*}$ & Main contribution ( $k$ ) \\
\hline 1 & 4.97 & $3.75(4 p)$ & 4.67 & $4.15(4 p)$ & 3.22 & $2.81(4 p)$ \\
\hline 2 & -16.06 & $-15.83(48)$ & -12.24 & $-11.13(4 s)$ & -8.33 & $-7.60(4 s)$ \\
\hline 3 & -16.06 & $-15.83(4 s)$ & -12.24 & $-11.13(48)$ & -8.33 & $-7.60(48)$ \\
\hline 4 & 242.82 & $239.20(3 d)$ & 54.22 & $50.44(3 d)$ & 30.70 & $26.81(3 d)$ \\
\hline 5 & 0.72 & $1.19(f-$ cont $)$ & 0.73 & 1.31 (f-cont) & 2.34 & $3.02(f-\operatorname{con} t)$ \\
\hline 6 & 0.72 & 1.19 (f-cont) & 0.73 & $1.31(f-\operatorname{con} t)$ & 2.34 & $3.02(f-\operatorname{con} t)$ \\
\hline 7 & $1.82-\mathrm{i} 95.68$ & $-j 95.68$ (d.cont) & $7.48-\mathrm{i} 86.06$ & $-\mathrm{i} 86.06$ (d-cont) & $36.75-\mathrm{i} 100.06$ & $-\mathrm{i} 100.06(\mathrm{~d}-\operatorname{con} \mathrm{l})$ \\
\hline 8 & $-4.40-i 0.17$ & $-3.05(4 p)$ & $-4.06-i 0.18$ & $-2.61(4 p)$ & $-3.64-10.19$ & $-2.27(4 p)$ \\
\hline 9 & $-4.40-i 0.17$ & $-3.05(4 p)$ & $-4.06-i 0.18$ & $-2.61(4 p)$ & $-3.64-\mathrm{i} 0.19$ & $-2.27(4 p)$ \\
\hline$M$ & $210.13-i 96.02$ & & $35.23-i 86.42$ & & $51.41-1100.44$ & \\
\hline$M_{\mathrm{sh}}$ & 207.54 & $(3 d, 4 s, 4 s)$ & 28.18 & $(3 d, 4 s, 4 s)$ & 11.61 & $(3 d, 4 s, 4 s)$ \\
\hline$M_{t s}$ & 0.0 & & 0.0 & & 0.0 & \\
\hline$M_{\mathrm{re}}$ & $-i 95.68$ & (d-cont) & $-i 86.06$ & (d-cont) & $-\mathbf{i} 100.06$ & (d-cont) \\
\hline
\end{tabular}

$-16.06$

50.44 (3d)

$-8.33$

$26.81(3 d)$

1.19 (f-cont)

$1.82-\mathrm{i} 95.68$

$-j 95.68$ (d-cont)

$7.48-\mathrm{i} 86.06$

$-4.06-i 0.18$

os (d-cont)

36.75-i100.06

$-3.64-10.19$

$-3.64-\mathrm{i} 0.19$

$-4,40-i 0.17$

$5.23-\mathrm{i} 86.4$

$-2.61(4 p)$

1.61

(d-cont) 
different points of the energy distribution: $\varepsilon_{1} \ll \varepsilon_{2}, \varepsilon_{1}=\varepsilon_{2}, \varepsilon_{1} \gg \varepsilon_{2}$ (note that the data in table 2 correspond to the case of $e_{1} \equiv e_{p}, e_{2} \equiv e_{d} ;$ in order to obtain the symmetrical distribution function, one would have to take into account the case of $e_{1} \equiv e_{\mathrm{d}}, e_{2} \equiv e_{\mathrm{p}}$ ). The table also shows the intermediate states $k$ which yield the main contributions to $M_{\alpha}$ and the model amplitudes according to equations (13), (15) and (16).

If $\varepsilon_{\mathrm{p}} \ll \varepsilon_{\mathrm{d}}$, the 'shake off' process dominates ( $\approx 80 \%$ of probability). Both the absolute and the relative 'shake off' contributions are decreasing with increasing p-electron energy. At the same time, the value of $M_{r c}$ does not vary much and becomes dominant when $\varepsilon_{\mathrm{p}} \geqslant \varepsilon_{\mathrm{d}}\left(=90 \%\right.$ of probability, if $\varepsilon_{\mathrm{p}} \gg \varepsilon_{\mathrm{d}}$ ).

This short analysis shows that the different model mechanisms of the transition dominate at different points of the electron energy distribution.

\subsection{Transition $3 d^{-1} 5 p\left[{ }^{\prime} P\right] \rightarrow 4 s^{-1} 4 p^{-1}\left[{ }^{1} P\right]+e_{1}+e_{2}$}

The main feature of this transition, as we have mentioned above, is that it may proceed by two steps due to the presence of the $4 s^{-2} 5 p$ state between the initial $3 d^{-1} 5 p\left[{ }^{1} P\right]$ and final ionic $4 s^{-1} 4 p^{-1}\left[{ }^{1} P\right]$ states. The first step is the Auger-like decay $3 d^{-1} 5 p \rightarrow$ $4 s^{-2} 5 p+e_{1}$ with the $5 p$-excited electron as a 'spectator', and the second step is the decay of the autoionization state $4 \mathrm{~s}^{-2} 5 \mathrm{p} \rightarrow 4 \mathrm{~s}^{-1} 4 \mathrm{p}^{-1}+e_{2}$, where now the $4 \mathrm{~s}$ vacancy is a 'spectator'. Of course this is not the only pathway of the transition $3 \mathrm{~d}^{-1} 5 \mathrm{p} \rightarrow$ $4 \mathrm{~s}^{-1} 4 \mathrm{p}^{-1}+e_{1}+e_{2}$. It may also proceed, similar to the one of section 4.2 , by simultaneous ejection of two electrons, which we shall call the 'direct' transition way in order to emphasize the difference from the two-step procedure.

The possibility of a two-step transition results in the appearance of two peaks in the electron spectrum in the energy regions around $29.27 \mathrm{eV}$ and $5.78 \mathrm{eV}$, which correspond to the ejection of the first- and the second-step electrons. The 'direct' transition leads to the ejection of so-called background electrons in the energy interval from $0 \mathrm{eV}$ to $35.05 \mathrm{eV}$.

According to the selection rules, the first-step electron may have the orbital angular momentum $1_{1}=2$, while the second-step electron orbital angular momentum is equal to zero or two. There is no such restriction for the orbital angular momenta of electron pairs ejected by 'direct' transition. The ejection of symmetrical $(1,1)$ and asymmetrical $(1,1+2), 1=0,1,2, \ldots$, electron pairs, which are coupled to ${ }^{1} S,{ }^{1} P$ and ${ }^{1} D$ terms, is possible in general.

In figure 4 we present the distribution functions $\gamma_{1,1}(\varepsilon)$ and $\gamma_{1,1+2}$ for the different electron pairs $(1,1)$ and $(1,1+2)$, which contribute to the transition $3 \mathrm{~d}^{-1} 5 \mathrm{p}\left[{ }^{1} \mathrm{P}\right] \rightarrow$ $4 s^{-1} 4 p^{-i}\left[^{i} P\right]+\left(e_{1}+e_{2}\right)\left[^{1} D\right]$. A similar behaviour of the distribution functions was calculated for transitions where the outgoing electrons are coupled to ' $\mathrm{S}$ and ' $\mathrm{P}$ terms, so that the corresponding figures have been omitted.

Similar to the transition $3 \mathrm{~d}^{-1} 5 \mathrm{p} \rightarrow 4 \mathrm{~s}^{-2}+e_{1}+e_{2}$, the 'shake-off' mechanism in the sense of section 3.1 is mainly responsible for the 'direct' decay $3 d^{-1} 5 p \rightarrow$ $4 s^{-1} 4 \mathrm{p}^{-1}+e_{1}+e_{2}$ at those energy regions where one electron is slow while the other is fast. But here the most interesting features are the peaks, which appear due to two-step transitions resulting in the ejection of $s$ and $d$ electrons. To calculate accurately the distribution functions at the peak regions, we used the experimental total width of the $4 s^{-2} 5 \mathrm{p}$ state, $\Gamma_{4 s^{-2} s p}=0.18 \mathrm{eV}$ (Sonntag 1992). Transitions $4 s^{-2} 5 \mathrm{p} \rightarrow 4 \mathrm{~s}^{-2} 4 \mathrm{p}^{-1}\left[{ }^{2,3} \mathrm{P}\right]+e$ with a $4 \mathrm{~s}$ vacancy as a 'spectator' mainly contribute to the total width of the $4 \mathrm{~s}^{-2} 5 \mathrm{p}$ state. According to our estimation using the simple HF 'spectator' approximation, the probabilities of these transitions are $\Gamma_{2}=0.109 \mathrm{eV}\left({ }^{1} \mathrm{P}\right)$ and $0.034 \mathrm{eV}\left({ }^{3} \mathrm{P}\right)$, so that the 


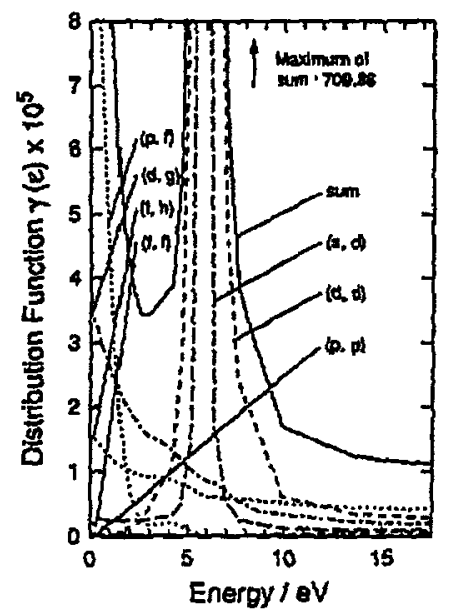

Figure 4. Lower part of the distribution functions $\gamma_{1, f}(\varepsilon)$ and $\gamma_{b_{1}+2}(\varepsilon)$ in the transition $3 d^{-1} 5 \mathrm{p}\left[{ }^{1} \mathrm{P}\right] \rightarrow$ $4 s^{-1} 4 p^{-2}\left[{ }^{1} P\right]+\left(e_{1}+e_{2}\right)\left[^{2} D\right]$ (left symmetrical half). The upper full curves corresponds to the total $Y^{\prime} D j(\varepsilon)$ of the above transition. The maximum value of the total $Y^{\prime} D_{3}(\varepsilon)$ is equal to $709.86 \times 10^{-5}$ at the resonance epergy $\varepsilon=5.78 \mathrm{eV}$.

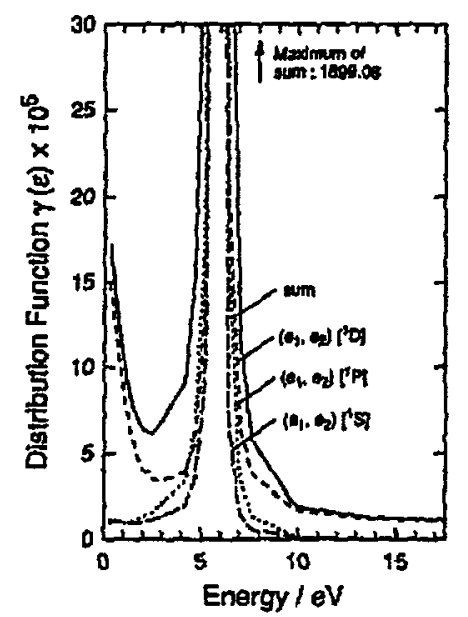

Figare 5. The total distribution function $\gamma_{10 x}(\varepsilon)$ (left symmetrical half, upper full curve) in the transition $3 d^{-1} 5 p\left[{ }^{\prime} P\right] \rightarrow 4 s^{-1} 4 p^{-1}\left[{ }^{1} P\right]+e_{1}+e_{2}$ with maximum value $1899.08 \times 10^{-5}$ at the resonance energy $\varepsilon=$ $5.78 \mathrm{eV}$. The contributions of the various electron pairs coupled to ' 5 , 'P and 'D terms are shown. The corresponding maxima at the same resonance energy amount to $370.32 \times 10^{-5}, 818.90 \times 10^{-5}$ and $709.86 x$ $10^{-5}$, respectively.

total value of $0.142 \mathrm{eV}$ is in reasonable agreement with the above experimental result. We also estimate the probability of the first-step transition $3 d^{-1} 5 p \rightarrow 4 s^{-2} 5 p+e$, using the same approach. The value obtained is $\Gamma_{1}=2.72 \times 10^{-3} \mathrm{eV}$.

Using these results, it is possible to approximately estimate the two-step probability of the transition $3 \mathrm{~d}^{-1} 5 \mathrm{p}\left[{ }^{-1} \mathrm{P}\right] \rightarrow 4 \mathrm{~s}^{-1} 4 \mathrm{p}^{-1}\left[{ }^{\prime} \mathrm{P}\right]+e_{1}+e_{2}$, assuming the independence of the steps, in the following way:

$$
\tilde{\Gamma}_{\mathrm{ts}}=\Gamma_{1}\left(\Gamma_{2} / \Gamma_{4 \mathrm{~s}^{-x_{\mathrm{P}}}}\right)=2.72 \times(0.109 / 0.142) \times 10^{-3} \mathrm{eV}=2.09 \times 10^{-3} \mathrm{eV} .
$$

The integral contributions of different electron pairs to the total probability of the transition $3 \mathrm{~d}^{-1} 5 \mathrm{p}\left[{ }^{1} \mathrm{P}\right] \rightarrow 4 \mathrm{~s}^{-1} 4 \mathrm{p}^{-1}\left[{ }^{1} \mathrm{P}\right]+e_{1}+e_{2}$ are listed in table 3. Figure 5 displays the distribution function $\gamma(\varepsilon)$ of the transition $3 d^{-1} 5 p\left[{ }^{1} P\right] \rightarrow 4 s^{-1} 4 p^{-1}\left[{ }^{1} P\right]+e_{1}+e_{2}$ as a sum of all $y_{3,1}(\varepsilon)$ and $y_{1,1+2}(\varepsilon)$. As can be seen in table 3 and figures 4 and 5 , the main contributions to the total probability of the transition under consideration are coming from the symmetrical electron pairs (d, d). The next important contribution stems from the $(s, d)$ pair. As was shown above, these electron pairs could be ejected only by two-step transitions. However, this does not mean that only the two-step process is responsible for the contribution of $(s, d)$ and $(d, d)$ electrons to the total probability. On the contrary, as one may see in figure 4 , it is difficult to separate the two-step and the 'direct' contributions. This separation is to some extent arbitrary. It could be done, for instance, by dividing the energy interval $(0, \Delta E)$ into at least three regions: $(a)$ $|\Delta \varepsilon| \leqslant \Gamma_{k} / 2,(b)|\Delta \varepsilon| \gg \Gamma_{k} / 2$ and $(c)$ the remaining part (here $\Delta \varepsilon$ and $\Gamma_{k}$ are defined according to equation (15) in the two-step transition model). Then the contribution of the electron pairs $(s, d)$ and $(d, d)$ to the total probability in the energy region $(a)$ 
Table 3. Integral contributions $\Gamma_{e_{r}=2}$ of different electron pairs $(\zeta C)$ and $(\zeta I+2)$ to the total transition probability of $3 \mathrm{~d}^{-1} \mathrm{Sp}\left[{ }^{1} \mathrm{P}\right] \rightarrow 4 \mathrm{~s}^{-1} 4 \mathrm{p}^{-\mathrm{t}}\left[{ }^{\mathrm{t}} \mathrm{P}\right]$ in $10^{-4} \mathrm{eV}$.

\begin{tabular}{|c|c|c|c|c|}
\hline $\begin{array}{l}\text { Electron pair } \\
\left(e_{1}, e_{2}\right)\end{array}$ & {$\left[L_{2} S_{2}\right]$} & $\Gamma_{e_{1, \times-2}}\left(10^{-4} \mathrm{eV}\right)$ & Fraction (\%) & \\
\hline $\begin{array}{l}(\mathrm{s}, \mathrm{s}) \\
(\mathrm{p}, \mathrm{p}) \\
(\mathrm{d}, \mathrm{d}) \\
\langle f, \mathfrak{f})\end{array}$ & $\begin{array}{l}\text { is } \\
\text { is } \\
\text { 's } \\
\text { is }\end{array}$ & $\begin{array}{r}0.029 \\
0.127 \\
25.711 \\
0.113\end{array}$ & $\begin{array}{r}0.1 \\
0.5 \\
98.9 \\
0.5\end{array}$ & \\
\hline$\Sigma \Gamma_{e_{1} e_{2}}$ & 's & 25.980 & 100.0 & 18.7 \\
\hline $\begin{array}{l}(p, p) \\
(d, d) \\
(f, f)\end{array}$ & $\begin{array}{l}1 p \\
{ }^{2} p \\
{ }^{2} p\end{array}$ & $\begin{array}{r}0.086 \\
56.182 \\
0.011\end{array}$ & $\begin{array}{r}0.2 \\
99.8 \\
0.0\end{array}$ & \\
\hline$\Sigma \Gamma_{a_{1} a_{2}}$ & 'p & 56.279 & 100.0 & 40.5 \\
\hline $\begin{array}{l}(\mathrm{s}, \mathrm{d}) \\
(\mathrm{p}, \mathrm{p}) \\
(\mathrm{p}, \mathrm{f}) \\
(\mathrm{d}, \mathrm{d}) \\
(\mathrm{d}, \mathrm{g}) \\
(\mathrm{f}, \mathrm{f}) \\
(\mathrm{f}, \mathrm{h})\end{array}$ & 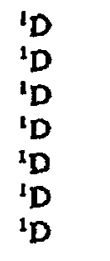 & $\begin{array}{r}5.203 \\
0.079 \\
2.036 \\
44.163 \\
2.765 \\
0.179 \\
2.309\end{array}$ & $\begin{array}{r}9.2 \\
0.1 \\
3.6 \\
77.8 \\
4.9 \\
0.3 \\
4.1\end{array}$ & \\
\hline$\Sigma \Gamma_{e, 2 z}$ & ${ }^{\mathrm{D} D}$ & 56.734 & 100.0 & 40.8 \\
\hline$\Sigma \Gamma_{e, 1,2}$ & & 138.993 & & 100.0 \\
\hline
\end{tabular}

$|\Delta \varepsilon| \leqslant \Gamma_{k} / 2$ around the resonance may be called 'two-step' transition probability. The contribution of the electrons $(\mathrm{s}, \mathrm{d})$ and $(\mathrm{d}, \mathrm{d})$ in the energy region $|\Delta \varepsilon| \gg \Gamma_{k} / 2$ and the contribution of 'non-two-step' electrons may be considered to represent the probability of the 'direct' transition. The remaining part of the total transition probability, which comes from the energy region where $\Delta \varepsilon$ and $\Gamma_{k}$ do not differ so much and $\Delta \varepsilon$ is comparatively small, has to be related to the interference of the two-step and direct transition channels.

So we may reasonably estimate the two-step probability of the transition $3 \mathrm{~d}^{-1} 5 \mathrm{p}[\mathrm{P}] \rightarrow 4 \mathrm{~s}^{-1} 4 \mathrm{p}^{-1}\left[{ }^{1} \mathrm{P}\right]+e_{1}+e_{2}$ to be approximately equal to $\Gamma_{\mathrm{s}}=$ $2 \Gamma_{4 s^{-2} s_{p}} y(\varepsilon=5.78)=6.82 \times 10^{-3} \mathrm{eV}$. This value reflects the influence of the many-body effects which lead to the increase of the previous approximate independent-two-step probability $\tilde{\Gamma}_{t s}=2.09 \times 10^{-3} \mathrm{eV}$ by a factor of about 3 . This result also demonstrates quite clearly the importance of the interelectron correlations in the atomic doubleionization processes.

\subsection{Transition $3 d^{-1} 5 p\left[{ }^{1} P\right] \rightarrow 4 s^{-1} 4 p^{-1}\left[{ }^{3} P\right]+e_{1}+e_{2}$}

The transition to the triplet doubly ionic state $4 \mathrm{~s}^{-1} 4 \mathrm{p}^{-1}\left[{ }^{3} \mathrm{P}\right]$ is similar to the previous one in many aspects. Of course, the released energy is larger, $\Delta E=37.895 \mathrm{eV}$ (we used the average value of the experimental energies $E_{\mathrm{P}_{\mathrm{f}}}=53.626 \mathrm{eV}, E_{\mathrm{P}_{1}}=53.358 \mathrm{eV}$ and $E_{\mathrm{P}_{2}}=52.930 \mathrm{eV}$ to estimate the released energy). Consequently, the second-step electron is ejected with an energy of $8.625 \mathrm{eV}$, while the first-step electron has, of course, the energy $29.27 \mathrm{eV}$, as in the transition to the singlet ionic state. 
Another difference lies in the fact that the total spin of an electron pair must be, according to the selection rules, equal to $S_{2}=1$, i.e. the outgoing electrons have parallel spins. This additional restriction leads to a significant mutual compensation of the partial amplitudes (equation (6)) and consequently to a much smaller value of the total amplitude.

The main numerical results for the transition $3 \mathrm{~d}^{-1} 5 \mathrm{p}\left[{ }^{1} \mathrm{P}\right] \rightarrow 4 \mathrm{~s}^{-1} 4 \mathrm{p}^{-1}\left[{ }^{3} \mathrm{P}\right]+e_{1}+e_{2}$ are presented in table 4 and figures 6 and 7 . The estimations of the two-step transition probabilities in the independent-two-step model and including interelectron correlations are equal to $\tilde{\Gamma}_{t s}=0.65 \times 10^{-3} \mathrm{eV}$ and $\Gamma_{t s}=2.21 \times 10^{-3} \mathrm{eV}$, respectively.

Summarizing the results of sections 4.1, 4.2 and 4.3 , we present in figures 8 and 9 the total distribution function $\gamma_{\text {tot }}(\varepsilon)$ as the sum of the symmetrical (in their own energy intervals) distribution functions of the transitions of sections 4.1, 4.2 and 4.3. As the energies of the above transitions are different, the resulting total distribution function is non-symmetrical.

The prominent peak $A$ in figures 8 and 9 corresponds to the ejection of the first-step electron in the transition $3 d^{-1} 5 p \rightarrow 4 s^{-2} 5 p+e$. Both the transitions $3 d^{-1} 5 p \rightarrow$ $4 \mathrm{~s}^{-1} 4 \mathrm{p}^{-1}\left[{ }^{1} \mathrm{P}\right]+e_{1}+e_{2}$ and $3 \mathrm{~d}^{-1} 5 \mathrm{p} \rightarrow 4 \mathrm{~s}^{-1} 4 \mathrm{p}^{-1}\left[{ }^{3} \mathrm{P}\right]+e_{1}+e_{2}$ have their peak values of $\gamma(\varepsilon)$ at energies around $29.27 \mathrm{eV}$. Only background electrons of the transitions of sections $4.13 \mathrm{~d}^{-1} 5 \mathrm{p} \rightarrow 4 \mathrm{~s}^{-2}+e_{1}+e_{2}$ and $4.33 \mathrm{~d}^{-1} 5 \mathrm{p} \rightarrow 4 \mathrm{~s}^{-1} 4 \mathrm{p}^{-1}\left[{ }^{3} \mathrm{P}\right]+e_{1}+e_{2}$, which are exclusively ejected due to the correlations, contribute to the peak $B$ of the transition of section $4.23 d^{-1} 5 p \rightarrow 4 s^{-1} 4 p^{-1}\left[{ }^{1} P\right]+e_{1}+e_{2}$, and vice versa. Therefore, the peaks $B$ and $C$, which correspond to the ejection of slow second-step electrons, have almost the same heights as in figures 5 and 7 , respectively.

Table 4. Integral contributions $\Gamma_{e t e z}$ of different electron pairs $(f l)$ and $(b l+2)$ to the total transition probability of $3 \mathrm{~d}^{-1} 5 \mathrm{p}\left[{ }^{1} \mathrm{P}\right]+4 \mathrm{~s}^{-1} 4 \mathrm{p}^{-1}\left[{ }^{3} \mathrm{P}\right]$, in $10^{-4} \mathrm{eV}$.

\begin{tabular}{|c|c|c|c|c|}
\hline $\begin{array}{l}\text { Electron pair } \\
\left(e_{1} e_{2}\right)\end{array}$ & {$\left[L_{2} S_{2}\right]$} & $r_{e_{1,0.7}}\left(10^{-4} \mathrm{eV}\right)$ & Fraction (\%) & \\
\hline $\begin{array}{l}(s, s) \\
(p, p) \\
(d, d) \\
(f, f)\end{array}$ & $\begin{array}{l}{ }^{3} \mathrm{~S} \\
{ }^{3} \mathrm{~s} \\
{ }^{3} \mathrm{~S} \\
{ }^{3} \mathrm{~S}\end{array}$ & $\begin{array}{r}0.003 \\
0.004 \\
12.708 \\
0.001\end{array}$ & $\begin{array}{r}0.0 \\
0.0 \\
99.9 \\
0.0\end{array}$ & \\
\hline$\Sigma \Gamma_{t_{1}, e_{2}}$ & ${ }^{3} \mathrm{~s}$ & 12.716 & 100.0 & 17.4 \\
\hline $\begin{array}{l}(p, p) \\
(d, d) \\
(f, n)\end{array}$ & $\begin{array}{l}{ }^{3} \mathbf{p} \\
{ }^{3} \mathbf{p} \\
{ }^{3} \mathrm{P}\end{array}$ & $\begin{array}{r}0.016 \\
28.741 \\
0.003\end{array}$ & $\begin{array}{r}0.0 \\
99.9 \\
0.0\end{array}$ & \\
\hline$\Sigma \Gamma_{e, 0,2}$ & ${ }^{3} \mathbf{P}$ & 28.760 & 100.0 & 39.4 \\
\hline $\begin{array}{l}(s, d) \\
(p, p) \\
(p, f) \\
(d, d) \\
(d, g) \\
(f, f) \\
(f, h)\end{array}$ & $\begin{array}{l}{ }^{3} \mathrm{D} \\
{ }^{3} \mathrm{D} \\
{ }^{3} \mathrm{D} \\
{ }^{3} \mathrm{D} \\
{ }^{3} \mathrm{D} \\
{ }^{3} \mathrm{D} \\
{ }^{3} \mathrm{D}\end{array}$ & $\begin{array}{r}7.502 \\
0.031 \\
1.222 \\
22.187 \\
0.457 \\
0.005 \\
0.181\end{array}$ & \begin{tabular}{r|}
23.8 \\
0.1 \\
3.9 \\
70.2 \\
1.4 \\
0.0 \\
0.6
\end{tabular} & \\
\hline$\Sigma \Gamma_{0,02}$ & ${ }^{3} \mathrm{D}$ & 31.585 & 100.0 & 43.2 \\
\hline$\Sigma \Gamma_{e 1,8}$ & & 73.061 & & 100.0 \\
\hline
\end{tabular}




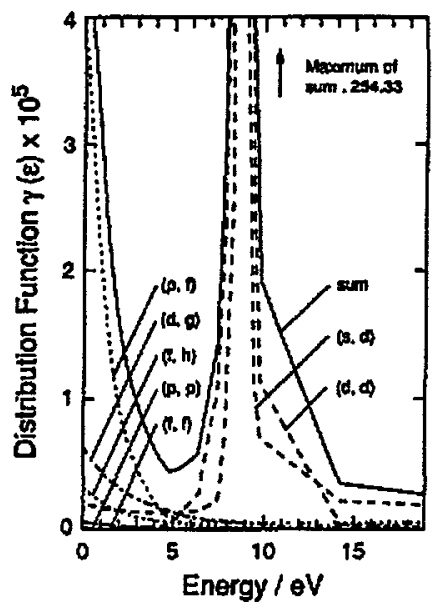

Figure 6. Lower part of the distribution functions $\gamma_{L,}(\varepsilon)$ and $\gamma_{L l+2}(\varepsilon)$ in the transition $3 \mathrm{~d}^{-1} 5 \mathrm{p}\left[{ }^{-1} \mathrm{P}\right] \rightarrow 4 \mathrm{~s}^{-1} 4 \mathrm{p}^{-1}\left[{ }^{3} \mathrm{P}\right]+\left(e_{1}+e_{2}\right)\left[^{3} \mathrm{D}\right]$ (left symmetrical haln). The upper full curve corresponds to the total $\left.\eta^{3}{ }^{3}\right](\varepsilon)$ of the above transition. The maximum value of the total $\left.\gamma^{3} \mathrm{D}\right](\varepsilon)$ is equal to $254.33 \times 10^{-5}$ at the resonance energy $z=8.62 \mathrm{eV}$.

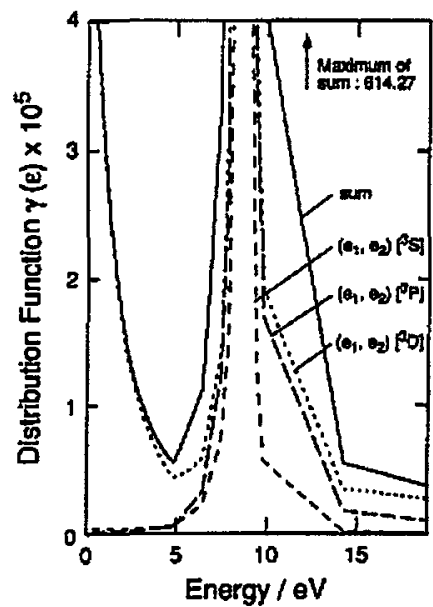

Figure 7. The total distribution function $\gamma_{\text {cot }}(\varepsilon)$ (left symmetrical half, upper full curve) in the transition $3 \mathrm{~d}^{-1} S \mathrm{p}[\mathrm{P}] \rightarrow 4 \mathrm{~s}^{-1} 4 \mathrm{p}^{-1}\left[^{3} \mathrm{P}\right]+e_{1}+e_{2}$ with maximum value $614.27 \times 10^{-5}$ at the resonance energy $\varepsilon=$ $8.62 \mathrm{eV}$. The contributions of various electron pairs coupled to ${ }^{3} \mathrm{~S},{ }^{3} \mathrm{P}$ and ${ }^{3} \mathrm{D}$ terms are also shown. The corresponding maxima at the same resonance energy amount to $110.62 \times 10^{-5}, 249.32 \times 10^{-5}$ and $254.33 \times$ $10^{-5}$, respectively.

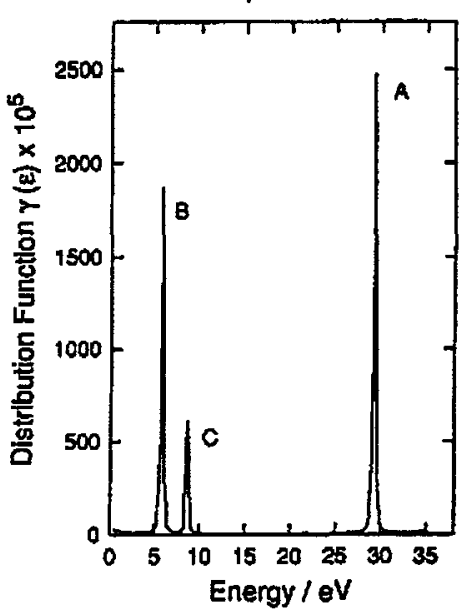

Figure 8. The total distribution function $\gamma_{\text {ka }}(\varepsilon)$ of the energy $e$ of an ejected electron, as a sum of the symmetrical (in their own energy transition interval) total distribution functions for the transitions from the initial $3 \mathrm{~d}^{-1} 5 \mathrm{p}\left[{ }^{1} \mathrm{P}\right]$ state to the final double-ion configurations $4 s^{-2}[1 S], \quad 4 s^{-1} 4 p^{-1}\left[{ }^{1} P\right]$ and $4 s^{-1} 4 p^{-1}\left[{ }^{3} \mathrm{P}\right]$. 


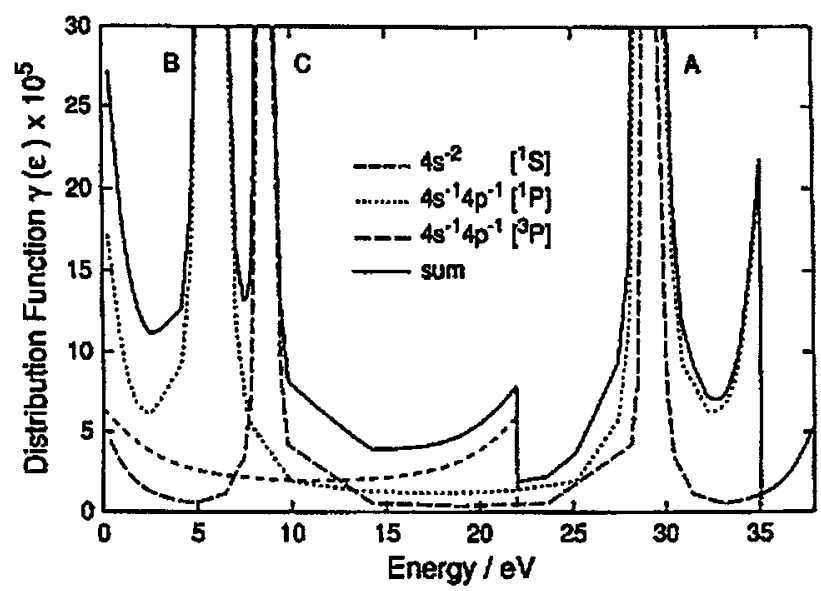

Flgare 9. Magnified lower part of figure 8, with symmetrical components well resolved.

\section{Final remarks}

The above results have been obtained by simplifying the real situation mainly in two respects, i.e. (i) by neglecting certain possible channels of autoionization and (ii) by using approximative calculation procedures.

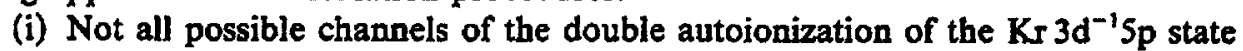
were considered in the present work, particularly the transition $3 d^{-1} 5 p\left[{ }^{1} \mathrm{P}\right] \rightarrow$ $4 \mathrm{p}^{-2}\left[{ }^{\top} \mathrm{S},{ }^{3} \mathrm{P},{ }^{1} \mathrm{D}\right]+e_{1}+e_{2}$ has been omitted. The final state may be populated also by two-step transitions, which would result in the appearance of additional peak structure in the electron spectra. Of course, a number of peaks additional to ours in figure 8 appear in the real spectrum due to the various 'up' and 'down' single autoionization transitions, such as $3 \mathrm{~d}^{-1} n l \rightarrow f_{1} f_{2} n^{\prime} l^{\prime}+\mathrm{e}$, which were outside the scope of the present work, too. Finally, the splitting of both initial and final states into the fine-structure components complicates the electron spectrum further. Peak $C$ in figure 8, e.g., splits into the components $C_{\mathrm{P}_{0}}, \mathrm{C}_{\mathrm{P}_{1}}$ and $\mathrm{O}_{\mathrm{P}_{2}}$, which are approximately proportional to the statistical weights $W_{\mathrm{P}_{0}}=\frac{1}{9}, W_{\mathrm{P}_{1}}=\frac{3}{9}$ and $W_{\mathrm{P}_{2}}=\frac{5}{9}$, due to the spin-orbital interaction.

(ii) The results represented in this work and the above discussion are based on the single-determinant HF approximation, which leads to the simplified energy level scheme of figure 1. The important effect, which is not taken into account here, is the configurational mixing of the states under consideration with the energetically adjacent ones. The configuration interaction may result not only in the shifts of peaks and variation of their intensity distribution, but also in the appearance of an unexpected peak structure. For example, one may suppose that the weak peak observed in the low energy region of the photoelectron spectrum (Lablanquie and Morin 1991) is due to the mixing of the intermediate $4 s^{-2} 5 p$ state with the $4 p^{-3} 4 d 5 p$ configuration. One of the mixed states has an energy somewhat higher than $4 s^{-2}$, so that the transition $3 d^{-1} 5 p \rightarrow$ $4 s^{-2}+e_{1}+e_{2}$ may proceed via this mixed state by two steps also.

Experimentally it is possible to distinguish the final states of the decay, namely $4 s^{-2}$ from $4 s^{-1} 4 p^{-1}$ and $4 p^{-2}$, by photon-induced fluorescence spectroscopy or photoelectron spectroscopy. These measurements are sensitive to the final-state configuration mixing which may be roughly estimated as follows. The relative intensity of a pure state $i$ in a complicated mixture of many states is given by the so-called spectroscopic 
factor $F_{i}$, which is known to be about 0.5 for the $4 \mathrm{~s}^{-1}$ state (Fuss et al 1981). In view of a comparison of our theoretical results with experimental ones, this can be taken into account simply by multiplying the cross sections derived without this effect by $F_{i}$. We did not calculate the spectroscopic factors of $4 \mathrm{~s}^{-2}, 4 \mathrm{~s}^{-1} 4 \mathrm{p}^{-1}$ and $4 \mathrm{p}^{-2}$, which is a separate and non-trivial task. However, in order to give a rough estimate of their role the following approximations were assumed: $F_{48}-2 \simeq\left(F_{48}-1\right)^{2}, F_{48}-s_{4 p^{-1}} \simeq F_{48}-1 F_{4 p} p^{-1}$ and $F_{4 p^{-2}} \simeq F_{4 p^{-1}} F_{4 p^{-1}}$. Using these simple relations and $F_{4 p^{-1}} \simeq 1$, it is concluded that in order to compare with experiment, the cross sections determined by the amplitudes shown in figure 1 must be multiplied by about 0.25 for $4 s^{-2}$ and by about 0.5 for the $4 \mathrm{~s}^{-1} 4 \mathrm{p}^{-1}$ states. However, one has to keep in mind that these approximate considerations may indicate the general tendency only and that the term dependence of $F_{i}$ (Aksela et al 1984) neglected here will complicate matters further.

\section{Conclusions}

Perturbation theory in the lowest non-vanishing order in interelectron interaction has been applied to the theoretical investigation of double-ionization decays of resonantly excited single-electron states. The formulae for the transition probabilities were derived in the $L S$ coupling scheme, and the orbital angular and spin momenta selection rules were obtained. Three approximate expressions, which correspond to illustrative model mechanisms of the transition, were derived as limiting cases of the exact ones.

The numerical results obtained for the decay of the resonantly excited $\mathrm{Kr} 13 \mathrm{~d}^{-1} 5 \mathrm{p}\left[{ }^{1} \mathrm{P}\right]$ state have demonstrated quite clearly the important role of the interelectron interactions in double-ionization processes.

On the other hand, the results presented show that low-energy electrons can appear in the photoelectron spectrum below the ionization threshold of the $3 d$ shell. As a function of photon frequency $\omega$, the yield of these low-energy electrons is strongly amplified by the resonant transition of the $3 \mathrm{~d}$ electron to $5 \mathrm{p}$ (or to other discrete levels), acting as an intermediate state, when $\omega$ approaches the transition energy.

We intend to study also the direct double-electron ionization amplitude in order to obtain not only the partial widths of the transitions under consideration, but also the total photoionization cross section in the discrete-excitation frequency region as a function of frequency. Note, that as a function of photon frequency the double-electron photoionization cross section is strongly enhanced when the process goes via the almost real discrete excitation of an inner-shell electron, like the $3 d \rightarrow 5 p$ transition.

The role of a 'doorway' discrete excitation ( $3 d^{-1} 5 p$ in our case) in amplifying the subsequent double-electron ionization can be played by any powerful resonances which persist in the photoabsorption spectrum. Namely, in the giant resonance region of Xe $(4 \mathrm{~d} \rightarrow \varepsilon f)$ or $\mathrm{Mn}(3 p \rightarrow 3 d)$ transitions, the probability of double-electron ionization of the outer shell must be strongly enhanced.

\section{Acknowledgment}

The authors would like to thank Professor B Sonntag and Dr E von Raven for communicating experimental data prior to publication. MA is grateful to the Alexander von Humboldt-Stiftung which made his stay and research in Germany possible. VK gratefully acknowledges the support of Professors L Chernysheva and I Lee in an early 
stage of the work and the continuous technical help by Mr B Baser, Mr S Luiz and Mr A Schuch during the computations at the Department of Physics, University of Kaiserslautern. This work has been funded by the Gcrman Federal Minister for Research and Technology under contract no 05452 AXI 5.

\section{Appendix}

Here the final formulae for the partial amplitudes (6) of the transition (in $\rightarrow f_{1} f_{2}+e_{1}+e_{2}$ ) are presented. To obtain them, the validity of $L S$ coupling scheme according to equation (9) of the main text is assumed. These formulae are:

$$
\begin{aligned}
& M_{1}=\oint_{n_{k}} \sum_{l_{k} \lambda_{1} \lambda_{2}}(-1)^{L_{1}+l_{f_{3}}+l_{n}+\lambda_{1}} \hat{L}_{1} \hat{L}_{2}\left\{\begin{array}{lll}
L & l_{2} & l_{k} \\
\lambda_{1} & l_{i} & l_{n}
\end{array}\right\}\left\{\begin{array}{lll}
l_{k} & l_{1} & L_{1} \\
l_{f_{2}} & l_{f_{1}} & \lambda_{2}
\end{array}\right\}\left\{\begin{array}{lll}
l_{2} & l_{k} & L \\
L_{1} & L_{2} & l_{1}
\end{array}\right\}
\end{aligned}
$$

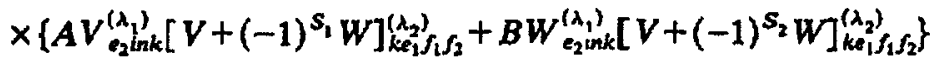

$$
\begin{aligned}
& \times E_{k \ell_{1} f_{1} f_{2}}^{-1} \delta_{L L^{\prime}} \delta_{M M^{*}} \\
& M_{2}=f_{n_{k} l_{k} \lambda_{2} \lambda_{2}}(-1)^{L_{2}+l_{k}+\lambda_{1}} \hat{L}_{1} \hat{L}_{2} \sum(-1)^{x} \hat{x}^{2}\left\{\begin{array}{lll}
L & l_{1} & x \\
\lambda_{1} & l_{1} & l_{m}
\end{array}\right\}\left\{\begin{array}{lll}
\lambda_{2} & l_{1} & x \\
\lambda_{1} & l_{f_{2}} & l_{k}
\end{array}\right\}
\end{aligned}
$$

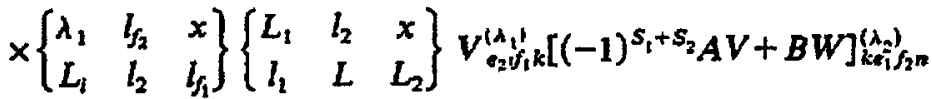

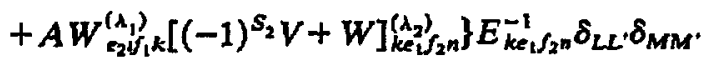

$$
\begin{aligned}
& M_{3}=\oint_{n_{k}} \sum_{k \lambda_{1} \lambda_{2}}(-1)^{l_{1}+l_{l_{2}}+\lambda_{2}+\lambda_{2}} \hat{L}_{1} \hat{L}_{2}\left\{\begin{array}{ccc}
L & \lambda_{1} & \lambda_{2} \\
l_{k} & l_{n} & l_{i}
\end{array}\right\} \sum_{x} \hat{x}^{2}\left\{\begin{array}{ccc}
L & \lambda_{1} & \lambda_{2} \\
I_{1} & l_{f_{1}} & x
\end{array}\right\}
\end{aligned}
$$

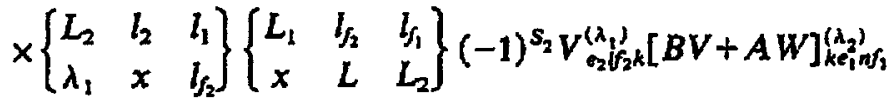

$$
\begin{aligned}
& \left.+(-1)^{S_{1}} A W_{e_{2} J_{3} k}^{\left.\lambda_{1}\right)}\left[V+(-1)^{S_{2}} W\right]_{k e_{1} n f_{1}}^{\left(\lambda_{1}\right)}\right\} E_{k e_{1} n f_{1}}^{-1} \delta_{L L} \cdot \delta_{M M} \text {. } \\
& M_{4}=f_{n_{k} l_{k} \lambda_{2} \lambda_{k}}(-1)^{L+l_{1}+L_{2}+l_{\delta_{2}}+l_{1}} \frac{\hat{L_{1}} \hat{L}_{2}}{\hat{L}_{2}}\left\{\begin{array}{ccc}
L_{1} & L_{2} & L \\
l_{1} & l_{k} & l_{2}
\end{array}\right\}\left\{\begin{array}{lll}
L_{1} & l_{f_{1}} & l_{\delta_{2}} \\
\lambda_{2} & l_{2} & l_{k}
\end{array}\right\} \delta_{\lambda_{1} L}
\end{aligned}
$$

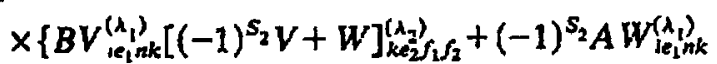

$$
\begin{aligned}
& \times\left[V+(-1)^{\left.\left.s_{1} W\right]_{k e_{2}}^{\left(\lambda_{2}\right)} \delta_{1}\right\}}\right\} E_{k \varepsilon_{2} f_{1} / \delta_{2}}^{-1} \delta_{L L^{\prime}} \delta_{M M^{\prime}} \\
& M_{5}=\oint_{n_{\lambda} l_{k} \lambda_{1} \lambda_{2}}(-1)^{l_{1}+l_{f_{1}}+l_{2}+\lambda_{1}} \hat{L}_{1} \hat{L}_{2} \sum_{x y}(-1)^{x} \hat{x}^{2} \hat{y}^{2}\left\{\begin{array}{lll}
l_{1} & L_{1} & x \\
L & l_{2} & L_{2}
\end{array}\right\}\left\{\begin{array}{lll}
x & L_{1} & l_{2} \\
l_{n} & \lambda_{2} & l_{i}
\end{array}\right\}
\end{aligned}
$$

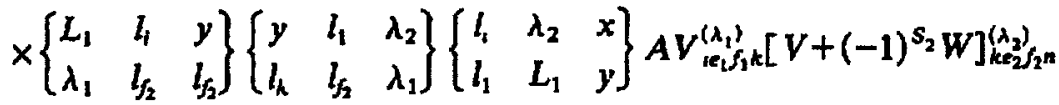

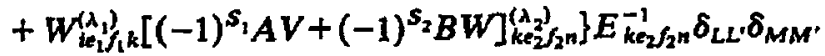

$$
\begin{aligned}
& M_{6}=\oint_{n_{k} l_{k} \lambda_{1} \lambda_{2}}(-1)^{L+L+l_{n}+l_{k}+\lambda_{1}} \hat{L}_{1} \hat{L}_{2} \sum_{x y}(-1)^{y} \hat{x}^{2} \hat{y}^{2}\left\{\begin{array}{ccc}
l_{1} & L_{1} & x \\
L & l_{2} & L_{2}
\end{array}\right\}\left\{\begin{array}{ccc}
l_{j_{2}} & l_{1} & y \\
l_{k} & l_{1} & \lambda_{1}
\end{array}\right\} \\
& \times\left\{\begin{array}{ccc}
y & \lambda_{2} & L \\
l_{n} & l_{i} & l_{k}
\end{array}\right\}\left\{\begin{array}{ccc}
l_{1} & L_{1} & x \\
l_{h_{1}} & y & l_{2}
\end{array}\right\}\left\{\begin{array}{ccc}
y & l_{f_{1}} & x \\
l_{2} & L & \lambda_{2}
\end{array}\right\}
\end{aligned}
$$

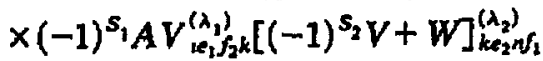

$$
\begin{aligned}
& \left.+W_{k_{1} \delta_{2} k}^{\left(\lambda_{1}\right\}}[B V+A W]_{k e_{2} n \delta_{2}}^{\left(\lambda_{1}\right)}\right\} E_{k e_{2}+f_{1}}^{-1} \delta_{L L^{\prime}} \delta_{M M^{\prime}}
\end{aligned}
$$




$$
\begin{aligned}
& M_{7}=\oint_{n_{k} l_{k} \lambda_{2} \lambda_{2}}(-1)^{L+L_{f_{2}}+l_{2}} \hat{L}_{1} \hat{L}_{2}\left\{\begin{array}{ccc}
L_{1} & l_{t} & l_{k} \\
l_{n} & L_{2} & L
\end{array}\right\}\left\{\begin{array}{lll}
L_{1} & l_{l} & l_{k} \\
\lambda_{2} & l_{l_{1}} & l_{2}
\end{array}\right\}\left\{\begin{array}{lll}
L_{2} & l_{n} & l_{k} \\
\lambda_{1} & l_{2} & l_{1}
\end{array}\right\} \\
& \left.\times A(-1)^{s_{2}} V_{e_{1} e_{2} n k}^{\left(\lambda_{1}\right)}\left[(-1)^{s_{1}} V+W\right]_{k l f_{1} f_{2}}^{\left(\lambda_{1}\right)}+W_{e_{1} e_{2} n k}^{\left(\lambda_{1}\right)}\left[(-1)^{s_{1}} V+W\right]_{k i f_{1} f_{2}}^{\left(\lambda_{2}\right)}\right] \\
& \times E_{k i f_{1}, \delta_{2}} \delta_{L L}, \delta_{M M} \\
& M_{8}=f_{n_{k}} \sum_{l_{4} \lambda_{1} \lambda_{2}}(-1)^{L_{1}+L_{2}+l_{1}+l_{n}+\lambda_{2}} \frac{\dot{\hat{L}_{1}} \hat{L}_{2}}{\hat{L}_{2}}\left\{\begin{array}{ccc}
L_{2} & \lambda_{2} & L_{1} \\
l_{f_{2}} & l_{1} & l_{h}
\end{array}\right\}\left\{\begin{array}{lll}
L_{2} & l_{1} & l_{2} \\
\lambda_{1} & l_{k} & l_{f_{1}}
\end{array}\right\} \delta_{\lambda_{2} L}
\end{aligned}
$$

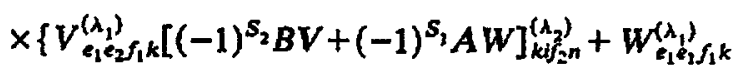

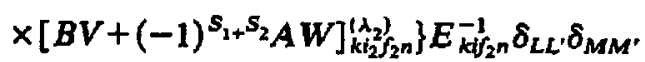

$$
\begin{aligned}
& M_{9}=f_{n_{k}} \sum_{l_{k} \lambda_{1} \lambda_{2}}(-1)^{L_{2}+\lambda_{2}+l_{1}+l_{2}} \hat{L}_{1} \hat{L}_{2}\left\{\begin{array}{ccc}
L & l_{k} & l_{i_{1}} \\
\lambda_{2} & l_{1} & l_{n}
\end{array}\right\}\left\{\begin{array}{lll}
L & l_{k} & l_{f_{1}} \\
l_{h_{2}} & L_{1} & L_{2}
\end{array}\right\}\left\{\begin{array}{lll}
L_{2} & l_{k} & l_{j_{2}} \\
\lambda_{1} & l_{1} & l_{2}
\end{array}\right\} \\
& \times\left\{V_{e_{1} e_{2} f_{2} k}^{\left(\lambda_{1}\right)}[A V+B W]_{k i n f_{1}}^{\left(\lambda_{2}\right)}+(-1)^{S_{2}} W_{e_{1} \varepsilon_{2} j_{2} k}^{\left(\lambda_{1}\right)}[A V+B W]_{k i n s}^{\left(\lambda_{2}\right\}}\right\} \\
& \times E_{k m f_{1}}^{-1} \delta_{L L}, \delta_{M M}
\end{aligned}
$$

where $A$ and $B$ are the spin factors, defined by expressions:

$$
\begin{aligned}
& A=(-1)^{S} \hat{S}_{1} \hat{S}_{2}\left\{\begin{array}{ccc}
\frac{1}{2} & \frac{1}{2} & S \\
S_{2} & S_{1} & \frac{1}{2}
\end{array}\right\} \delta_{S S^{\prime}} \delta_{M_{s}} M_{s}^{\prime} \\
& \left.B=(-1)^{S_{\mathrm{t}} \sqrt{2} \hat{S}_{2}\left\{S_{1}\right.} \frac{1}{2} \quad \frac{1}{2}\right\} \delta_{S_{1} S_{2}} \delta_{S 0} \delta_{S S^{\prime}} \delta_{M_{3} M_{3}^{\prime}}
\end{aligned}
$$

while the reduced direct and exchange Coulomb matrix elements are given by the formulae:

$$
\begin{aligned}
& V_{1324}^{(\lambda)}=\hat{l}_{1} \hat{l}_{2} \hat{l}_{3} \hat{l}_{4}\left(\begin{array}{lll}
l_{1} & \lambda & l_{2} \\
0 & 0 & 0
\end{array}\right)\left(\begin{array}{lll}
l_{3} & \lambda & l_{4} \\
0 & 0 & 0
\end{array}\right)\left\langle\nu_{1} \nu_{3}\left|\frac{r_{<}^{\prime}}{r_{>}^{l+1}}\right| \nu_{2} \nu_{4}\right\rangle \\
& W_{1324}^{(\lambda)}=\hat{\lambda} \sum_{j}(-1)^{j+j}\left\{\begin{array}{lll}
l_{1} & l_{2} & \lambda \\
l_{2} & l_{4} & j
\end{array}\right\} V_{1342 .}^{(j)} .
\end{aligned}
$$

Here the usual notations for $3 j m$ - and $6 j$-Wigner coefficients with the phase factors defined according to Warshalovich et al (1975), are used: $\hat{l}=(2 I+1)^{1 / 2}$.

The conditions, under which $3 j m-, 6 j$-Wigner coefficients and $\delta$-Kronecker symbols in (A.1-13) are not equal to zero, establish the orbital angular and spin momenta selection rules both for single-electron states, involved in the transition, and intermediate-coupling momenta $L_{2} S_{1}, L_{2} S_{2}$.

\section{References}

Aksela H, Aksela S and Pulkkinen H 1984 Phys. Reu. A 30 2456-61

Aksela H, Aksela S, Pulkkinen H, Bancroft G M and Tan K H 1986a Phys. Reu. A 33 3867-75

1986b Phys. Rev. A 33 3876-84

Aksela H, Aksela, S, Pulkkinen H and Yagishita A 1989 Phys. Rev. A $406275-80$

Amusia M Ya and Cherepkov N A 1975 Case Studies At. Phys. 5 47-121

Amusia M Ya, Kilin V A, Kolesnikova A N and Lee I S 1985 Pisma Zh. Tekn. Fiz 11343 ff (in Russian) Becker U, Hölzel R, Kerkhoff H G, Langer B, Szostak D and Wehlitz R 1986\& Phys. Reo. Lett. 56 1120-3 Becker U, Langer B, Kerkhoff H G, Kupsch M, Szostak D, Wehlitz R, Heimann P A, Lin S H, Lindle D W, Feret T A and Shirley D A 1988 Phys. Reo. Lett. 60 1490-3

Becker U, Prescher $T$, Schmidt E, Sonntag B and Wetzel H E 1986b Phys. Reu, A 33 3891-9 
Chernysheva L V and Amusia M Ya 1983 Automatization System for Atomic Structure Researches (Leningrad: Nauka) (in Russian)

Eberhardt W, Kalkoffen G and Kunz C 1978 Phys. Reu. Lett. 41 156-9

Ehresmann A, Vollweiler F, Wildberger $M_{3}$ Schmoranzer H, Möbus B and Schartner K H 1922 Proc 4 th Eur. Conf, on Atomic and Molecular Physics (Europhys. Conf. Abstracts 16B) ed K Bethge p 183

Fuss 1, Glass R, McCarthy I E, Minchinton A and Weigold E 1981 J. Phys. B: At. Mol. Phys. 14 3277-87

Hall R I, Avaldi L, Dawber G, Rutter P M, MacDonald A M and King G C 1989 J. Phys. B: At. Mol. Opt. Phys. 22 3205-16

Hall R I, Avaldi L, Dawber G, Zubek N and King G C 1990 J. Phys. B: At. Mol Opt. Phys. 23 4469-85

Hall R I, Eltis K, McConkey A, Dawber G, Avaldi L, MacDonald M A and King G C 1992 J. Fhys. B: At. Mol. Opt. Phys. 25 377-88

Lablanquie P and Morin P 1991 J. Phys. B: At. Mol. Opt. Phys. 24 4349-62

Lindle D W, Heimann P A, Ferret T A, Piancastelli M N and Shirley D A 1987 Phys. Reu A $354605-10$

Moore C E 1971 Atomic Energy Levels (NBS Reprint from Circular No 467) (Washington DC: US Govt Printing Office)

Schartner K H, Lenz P, Mōbus B, Schmoranzer H and Wildberger M 1989 J. Phys. B: At. Mol. Opt. Phys. 22 1573-81

Schartner K H, Magel B, Mäbas B, Schmoranzer H and Wildberger M $1990 \mathrm{~J}$. Phys. B: Al. Mol. Opt. Phys. 23 LS27-32

Schartner K H, Möbus B, Lenz $P_{2}$ Schmoranzer H and Wildberger M 1988 Phys. Rev. Lett $612744-7$

Schmoranzer H, Wildberger M, Ehresmann A, Magel B, Möbus B and Schartner K H 1991 BESSY Annual Report pp 108-9

Schmoranzer H, Wildberget M, Schartner K H, Möbus B and Magel B 1990 Phys. Letc. 150A 281-5

Sonntag B 1992 Private communication

Svensson S, Eriksson B, Martensson N, Wendin G and Gelius N 1988 J. Electron Spectrose Relat. Phenom. 47 327-84

von Raven E, Meyer M, Pabler M and Sonntag B $1990 \mathrm{~J}$. Electron Spectrasc, Relat. Phenom 52 677-88

Warchalovich D A, Moskalev A N and Ersonskij V K 1975 Quentum Theory of Angular Momentum (Leningrad: Nauka) (in Russian)

Wills A A, Cafolla A A and Comer J 1990a J. Phys. B: At. MoL. Opt. Phys. 23 2029-36

Wills A A, Cafolla A A, Svensson A and Comer I 1990b J. Phys. B: At. MoL Opt. Phys. 23 2013-28 